\title{
Qualidade de revistas científicas: uma revisão sistemática da literatura
}

\author{
Mariane Sperber \\ Universidade Federal de Santa Catarina, Departamento da Ciência da Informação, Florianópolis, SC, \\ Brasil \\ sperbermari@gmail.com \\ Vinicius Medina Kern \\ Universidade Federal de Santa Catarina, Departamento da Ciência da Informação, Florianópolis, SC, \\ Brasil \\ v.m.kern@ufsc.br
}

DOI: https://doi.org/10.26512/rici.v12.n3.2019.21100

Recebido/Recibido/Received: 2018-12-20

Aceitado/Aceptado/Accepted: 2019-08-08

ARTIGOS DE REVISÃo

Resumo: A avaliação da atividade científica é um fato que tem crescido e sido bastante evidenciado na comunidade acadêmica nas últimas décadas. As revistas científicas, principais fontes de informação na atualidade e responsáveis pela difusão do conhecimento por meio de publicações científicas, são os meios de avaliação da atividade científica mais visionados. Em meio a era digital e a necessidade da publicação para a validação dos estudos, é eminente o aumento da produção científica, que consequentemente acarreta o surgimento de novos títulos de revistas. O surgimento destes novos títulos esparra na necessidade da avaliação das revistas científicas. Acredita-se que seja necessário propor parâmetros e indicadores de qualidade que auxiliem nesta avaliação. Como sabe-se, já existem indicadores de qualidade que auxiliam na avaliação das revistas científicas, dos quais o fator de impacto e o Qualis Capes são os mais utilizados e enfatizados na literatura. Mas até que ponto podemos considerar o fator de impacto e o Qualis Capes como os principais indicadores de qualidade das revistas científicas e utilizá-los em grande maioria para classificar a qualidade de uma revista científica? Partindo dos pontos supracitados, a pesquisa em questão pretende realizar uma análise da literatura, mais especificamente uma revisão sistemática da literatura a fim de responder a seguinte questão: o que caracteriza uma revista científica de qualidade de acordo com a literatura científica? De antemão, pode-se concluir que a literatura ainda está muito restrita à qualidade de revistas científicas, utilizando como principal indicador o fator de impacto, mas outros indicadores não menos importantes também estão ganhando espaço. Percebe-se ainda que é um tema incipiente, apesar da grande preocupação dos autores de artigos em publicarem seus manuscritos em revistas de renome.

Palavras-chave: Revista científica. Qualidade de revista científica. Fator de Impacto. Indicadores de qualidade.

\section{Calidad de revistas científicas: una revisión sistemática de la literatura}

Resumen: La evaluación de la actividad científica es un hecho que ha crecido y ha sido bien evidenciado en la comunidad académica en las últimas décadas. Las revistas científicas, las principales fuentes de información en la actualidad y responsables de la difusión del conocimiento a través de publicaciones científicas, son los medios para evaluar la actividad científicas más vistas. En medio de la era digital y la necesidad de publicación para la validación de estudios, el aumento de la producción científica es inminente, lo que conlleva la aparición de nuevos títulos de revistas. La aparición de estos nuevos títulos se propaga en la necesidad de la evaluación de revistas científicas. Se cree que es necesario proponer 
parámetros e indicadores de calidad para ayudar en esta evaluación. Como se sabe, ya existen indicadores de calidad que ayudan en la evaluación de revistas científicas, de las cuales el factor de impacto y los Qualis Capes son los más utilizadas y destacados en la literatura. Pero ¿hasta qué punto podemos considerar el factor de impacto y los Qualis Capes como los principales indicadores de calidad de las revistas científicas y utilizarlos en gran mayoría para clasificar la calidad de una revista científica? Sobre la base de los puntos antes mencionados, la investigación en cuestión pretende realizar un análisis de la literatura, más específicamente una revisión sistemática de la literatura para responder la siguiente pregunta: ¿qué caracteriza a una revista científica de calidad según la literatura científica? De antemano, se puede concluir que la literatura todavía está muy restringida a la calidad de las revistas científicas, utilizando como indicador principal el factor de impacto, pero otros indicadores no menos importantes también están ganando espacio. También se percibe como un tema incipiente, a pesar de la gran preocupación de los autores por los artículos que publican sus manuscritos en revistas de renombre.

Palabras clave: Revista científica. Calidad de la revista científica. Factor de impacto. Indicadores de calidad.

\section{Quality of scientific journals: a systematic review of the literature}

Abstract: The evaluation of scientific activity is a fact that has grown and been well evidenced in the academic community in recent decades. Scientific journals, the main sources of information at the present time and responsible for the diffusion of knowledge through scientific publications, are the means of evaluating the scientific activity most viewed. During the digital age and the need for publication for the validation of studies, the increase in scientific production is imminent, which consequently entails the appearance of new journal titles. The emergence of these new titles spreads in the need of the evaluation of scientific journals. It is believed that it is necessary to propose parameters and quality indicators to assist in this evaluation. As is known, quality indicators already exist that aid in the evaluation of scientific journals, of which the impact factor and the Qualis Capes are the most used and emphasized in the literature. But to what extent can we consider the impact factor and the Qualis Capes as the main quality indicators of scientific journals and use them in large majority to classify the quality of a scientific journal. Based on the points, the research in question intends to carry out an analysis of the literature, more specifically a systematic review of the literature in order to answer the following question: what characterizes a scientific journal of quality according to the scientific literature? In advance, it can be concluded that the literature is still very restricted to the quality of scientific journals, using as main indicator the impact factor, but other indicators not less important are also gaining space. It is also perceived as an incipient subject, despite the great concern of authors of articles in publishing their manuscripts in renowned journals.

Keywords: Scientific journal. Quality of scientific journal. Impact factor. Quality indicators.

\section{Introdução}

Avaliar as atividades científicas é um fato que, nas últimas décadas vem sendo muito evidenciado no contexto da comunicação científica. Fato decorrente da grande quantidade de informações disponíveis nas mais diversas plataformas da Web. Outro ponto que merece destaque é a necessidade da publicação de pesquisas científicas para conferir validade científicas aos artigos científicos publicados, visto que pesquisas não disponibilizadas, não são conhecidas e reconhecidas, muito menos trazem contribuições para a comunidade científica.

O aumento das atividades de pesquisas e consequentemente a quantidade de artigos submetidos e publicados diariamente, acarreta o crescimento de novos títulos de revistas diariamente. Portanto para, conferir validade a estes títulos de revistas é necessário avaliar os aspectos de qualidade que compõe as mesmas. Krzyzanowski e Ferreira (1998) apontam uma preocupação constante dos profissionais da informação que se interessam pela qualidade da 
informação científica e a qualidade do conteúdo das revistas científicas, visto que a proliferação de novos títulos de revistas científicas é constante e crescente. Consideradas por Targino e Garcia (2008, p. 61), o "espelho da ciência", as revistas científicas são importantes instrumentos de difusão do conhecimento, desempenhando assim papel fundamental na comunidade científica.

O primeiro estudo datado sobre os aspectos e indicadores de qualidade das revistas científicas foi realizado no ano de 1964 pela Unesco, com a finalidade de criar um modelo para avaliar as revistas latino-americanas (Santos, 2010). Seguindo-se com os estudos de Arends (1968); Braga e Oberhofer (1982); Yahn (1985); Martins (1986); Krzyzanowski, Krieger e Duarte (1991); Castro e Ferreira (1996); Krzyzanowski e Ferreira (1998); Souza e Yamamoto (1999); Yamamoto et al. (2002), Ferreira e Krzyzanowski (2003), Alonso-Gamboa (2003), Barbalho (2005), Schultze (2005), Trzesniak (2006), dentre outros.

Como sabemos, atualmente os indicadores mais utilizados para a avaliação das revistas científicas no Brasil são o Fator de Impacto, que mede a qualidade por meio de citações dentro de determinado período de tempo e o Qualis Capes, que avalia as revistas científicas por meio de estratos, que vão do nível mais baixo $C$, ao nível mais alto $A 1$. Ou seja, grande parte das revistas científicas têm sido avaliadas quanto ao fator de impacto, que é uma medida peculiar baseada em base de dados específica, janela de tempo específica, problemas de mensuração que são apontados na literatura, são índices "negociados". Porém, o Fator de Impacto é só uma medida de "impacto" em termos de citações. E qualidade, o que é?

Percebe-se que a literatura sobre qualidade de revistas científicas muito enfatiza os indicadores baseados em citações, em especial, o fator de impacto, o que é apenas um aspecto da qualidade de revista, mas é necessário analisar também outros aspectos não menos importantes contidos nas revistas. Partindo dos pontos supracitados, o estudo em questão pretende realizar uma revisão sistemática da literatura, com o intuito analisar a literatura científica em relação as principais discussões acerca da qualidade das revistas, bem como se possível elencar outros indicadores a partir do resultado da revisão. Para nortear os passos da RSL (Revisão Sistemática da Literatura), definiu-se a seguinte pergunta de pesquisa: o que é qualidade de revistas científicas no âmbito da comunicação científica?

Sendo assim, nas próximas seções, serão descritos os procedimentos metodológicos utilizados para a realização da pesquisa e os resultados por meio da aplicação dos critérios de inclusão dos artigos, apresentando-se assim, os dados obtidos após sua aplicação, bem como a discussão dos dados da pesquisa, conclusão e por fim as referências utilizadas para a compilação do estudo. 


\section{Procedimentos Metodológicos}

Para realizar a revisão sistemática da literatura em questão, foram percorridas as seguintes etapas: estabelecimento da pergunta de pesquisa e objetivos da RSL (Revisão Sistemática da Literatura); estabelecimento dos critérios de exclusão e inclusão dos artigos, ou seja, a seleção da amostra da pesquisa; a definição das informações que serão extraídas dos artigos selecionados após a utilização dos critérios de exclusão ou inclusão; análise dos dados extraídos, apresentação e discussão dos resultados, bem como os resultados da pesquisa.

Para selecionar os artigos a serem utilizados, formam selecionados três bases de dados específicas da Ciência da Informação: a BRAPCI (Base de Dados de periódicos da Ciência da Informação), por tratar-se de uma base de dados brasileira e por englobar publicações de estudos da Ciência da Informação no Brasil; Scopus, base de dados com publicações revisadas por pares, oferecendo abrangente produção sobre as pesquisas do mundo nas áreas de ciência, tecnologia, medicina, ciências sociais, artes e humanas; e a ScienceDirect, base de dados com publicações de mais de 2.500 revistas científicas nas áreas de Ciências Físicas e Engenharia, Ciências Biológicas, Ciências da Saúde e Ciências Sociais e Humanas, pertencente a editora Elsevier. Ambas as revistas internacionais apresentadas foram escolhidas por englobarem publicações a respeito da Ciência da Informação em sua coleção.

Os critérios de inclusão definidos para a revisão sistemática em questão foram: a seleção de artigos em português, inglês e espanhol, com resumos, palavras-chave e o texto completo em sua base, no período comprometido entre 2003 a 2018; e artigos que em seu contexto englobem ou abordem aspectos e conceitos que dizem respeito à qualidade das revistas científicas. $\mathrm{O}$ contexto dos artigos a serem selecionados diz respeito tanto a presença da menção de algum aspecto de qualidade no título, resumo e palavras-chave do artigo.

A busca nas bases de dados foi realizada de forma distinta, em virtude das características de acesso diferentes de cada base, sendo adotadas estratégias adaptadas para cada uma, de acordo com a delimitação da pergunta de pesquisa e dos critérios de seleção elencados para a revisão sistemática. A realização da busca foi feita por meio do acesso on-line, utilizando-se especificamente apenas um termo: "journal quality".

As palavras-chave consideradas nessa busca, por sua vez, foram: journal rankings. journal quality, impact factor, h-index, calidad de las revistas científicas, revistas científicas, revisão por pares, peer review.

Para a coleta de dados foram feitos formulários de condução da pesquisa, disponibilizados no Apêndice $A$, com a finalidade de extrair mais precisamente os artigos a serem selecionados. Nestes formulários de condução extraíram-se dados, tais como, data de busca, 
detalhamento da busca, palavras-chave utilizadas, strings de busca e por fim uma listagem de artigos incluídos e excluídos.

Após uma minuciosa leitura do título e dos resumos, aplicando-se os critérios de inclusão e exclusão, obtendo como amostra para a pesquisa 18 artigos. Com os resultados dos artigos obtidos, espera-se uma discussão que apresente dados concretos e importantes sobre a qualidade das revistas científicas e seus respectivos aspectos e/ou indicadores de qualidade.

\section{Resultados}

Na presente revisão sistemática da literatura, analisou-se 18 artigos que atenderam aos critérios de inclusão estabelecidos previamente. Dentre os artigos incluídos na revisão sistemática, cerca de mais da metade dos artigos incluídos falam sobre o fator de impacto como medida de qualidade de revistas científicas, evidenciando de antemão que é e ainda continua sendo o principal e mais conhecido indicador de qualidade de revistas. Pôde-se perceber também, que os artigos foram redigidos em sua grande maioria com coautoria, em que seus autores discutem a qualidade das revistas principalmente na área das Ciências Humanas. Três dos artigos, apresentam o método de avaliação em seus próprios países: Reino Unido, Indonésia e Índia.

Em relação às revistas em que se encontram os artigos incluídos, quatro dos selecionados, se encontram nas revistas: Ponto de Acesso e Encontros Bibli: Revista Eletrônica de Biblioteconomia e Ciência da Informação , Em Questão: revista da Faculdade de Biblioteconomia e Comunicação da UFRGS e os outros doze artigos, encontram-se nas revistas internacionais, a saber: European Journal of Operational Research; Journal of Informetrics; Critical Perspectives on Accounting; Scientometrics; Information Processing \& Management, Wiley Online Library; e Research Evaluation.

Quanto ao tipo de delineamento de pesquisa dos artigos incluídos, os estudos em sua grande maioria apresentam revisões da literatura, acerca dos indicadores de qualidade das revistas, excepcionalmente os indicadores bibliométricos, dos quais o fator de impacto é o indicador mais evidenciado, bem como estudos de caso, pesquisa exploratória, pesquisa de ação e pesquisa bibliográfica.

\section{Apresentação dos Dados}

Nesta seção serão descritos os dados dos documentos encontrados, com enfoque nos indicadores e aspectos da avaliação da qualidade das revistas científicas.

Desde modo, começamos a tratar do artigo de Carreli e Giannasi-Kaimen (2009), que apresenta um estudo sobre os aspectos intrínsecos das revistas científicas de Qualis A da Ciência 
da Informação. Os autores destacam de antemão, que a revista científica é uma fonte de informação que desempenha papel fundamental na comunidade científica e asseguram que sua qualidade deve ser mantida na transferência do formato impresso ao eletrônico. Dentre os aspectos analisados pelos autores em sua pesquisa, destacaram-se os dados gerais dos periódicos; o tempo de existência de cada título; a periodicidade dos periódicos científicos; a presença dos títulos nos mecanismos de indexação e resumos e em repositórios digitais; as seções de cada título; a equipe editorial; o sistema de organização; o sistema de navegação; o sistema de rotulagem; o sistema de busca; o conteúdo das informações; a usabilidade do site; e os tipos de documentos de comportam (html, pdf etc.).

Mingers e Yang (2017), acreditam que a avaliação da qualidade das revistas científicas está se tornando cada vez mais importante no desempenho do meio científico. Apontam que a análise de indicadores de qualidade é um dos estudos mais realizados para avaliar a qualidade das revistas científicas. Em seu estudo, destacam seis indicadores quantitativos de qualidade das revistas científicas, dos quais a avaliação dos pares e os indicadores de citação têm sido a forma principal de avaliar a qualidade das revistas. Discutem, também a questão do local de publicação de um artigo, ou seja, a revista científica, ponto muito destacado quando se trata de avaliação de revistas. Seu principal intuito deste modo, é avaliar os indicadores atualmente disponíveis em quatro critérios: precisão, robustez, transparência e imparcialidade, em que se observa que os principais indicadores de revistas científicas disponíveis na atualidade, do mesmo modo, são os principais utilizados na prática e decisão sobre listas de classificação de revistas científicas. Sendo assim, acreditam que, o uso inapropriado destas métricas pode distorcer a qualidade das medidas baseadas em parâmetros com medidas no índice médio de citações.

Haddway et al. (2016), apresentam uma revisão da literatura na qual são apresentadas três fontes de métricas das revistas científicas, a saber: o impacto normalizado por papel (SNIP), o impacto bruto por papel (RIP), o Journal Impact Factor (JIF) e o julgamento de especialistas, discutindo assim a avaliação das revistas científicas por meio dos indicadores bibliométricos. Segundo os autores, a revisão por pares tem sido o padrão de garantia de qualidade na pesquisa acadêmica. Mas, por ser inerentemente subjetiva e qualitativa, a confiabilidade da revisão por pares como método para avaliar a qualidade da pesquisa tem sido frequentemente questionada. As questões incluem vieses na seleção de revisores, a tendência dos avaliadores de avaliar de acordo com seus próprios interesses, conflitos de interesses e vieses na avaliação da pesquisa. Em meio a isso, os padrões bibliométricos, principalmente baseados em citações, ganharam força, visto que poderiam fornecer uma medida quantitativa mais objetiva e alternativa de qualidade de pesquisa, que não sofreria influência da revisão dos pares. 
Rowlinson et al. (2015), destacam uma visão geral do debate de rankings de revistas científicas, identificando quatro críticas em relação aos rankings do Guia ABS. A primeira das críticas apresentadas é a afirmação de que os revisores especialistas são capazes de discernir a qualidade dos artigos das revistas sem referência a métricas de citação ou rankings de revistas; a segunda, é o argumento, que discute as variações no nível de citações de artigos nos principais periódicos, significando que os rankings baseados em citações não são confiáveis como um guia para a qualidade de artigos individuais; a terceira, de acordo com o viés de campo da disciplina de crítica no Guia ABS sugere que uma lista de classificação geral para periódicos em negócios e gerenciamento é problemática; e, finalmente, os críticos acreditam que a construção de listas de revistas científicas leva a uma classificação de obsessão, que distorce o REF como uma auditoria de pesquisa cuja finalidade principal é a alocação de recursos, em vez de classificação.

Por sua vez, Lee e Shin (2014), discutem a medida de desempenho das revistas científicas, fato que tem sido motivo de preocupação para os formuladores de políticas científicas, bem como partes da academia, que consideram o fator de impacto como medida mais comumente utilizada no desempenho e avaliação da qualidade das revistas científicas. Apontam que o fator de impacto é sustentado pela suposição de que a frequência de citações mede com precisão a importância de uma revista para seus usuários finais. Decorrente de sua compreensibilidade, robustez, simplicidade e disponibilidade, o fator de impacto tem sido cada vez mais popular e amplamente utilizado para vários propósitos na comunidade acadêmica. Mas o fator de impacto também possui suas desvantagens e com elas as críticas. Apresentam quatro críticas sobre o FI: a representatividade, a cobertura, a definição operacional e dependência de campo. Em primeiro lugar, o $\mathrm{Fl}$ de uma revista científica não é estatisticamente representativo em artigos individuais publicados nas revistas; a segunda questão está associada a cobertura restrita do banco de dados usado para calcular o Fl, em que os bancos de dados têm preferência pelos artigos de língua inglesa e os livros nem sequer são incluídos nas fontes, embora uma fração substancial da produção científica seja publicada em forma de livros. Em terceiro lugar, o $\mathrm{Fl}$ apresenta alguns problemas técnicos com a sua definição operacional. O FI de uma revista é o número médio de citações recebidas por artigo publicado médio durante dois anos anteriores. Um dos problemas é que os tipos de artigos incluídos no numerador e o denominador não são consistentes, ou seja, somente itens citáveis, como artigos, notas e revisões, são incluídos no denominador, enquanto no numerador contém citações de todos os tipos de publicações, incluindo editoriais, cartas e resumos de reuniões. A composição dos tipos de artigos também é um fator que influencia no $\mathrm{Fl}$, uma vez que as revisões são mais prováveis de serem citadas do que os trabalhos de pesquisa originais. A quarta questão é a dependência de campo do $\mathrm{Fl}$, que é exatamente o que este estudo procura resolver. 
Miglioli (2017), em seu estudo afirma que o fator de impacto é o índice padrão de medição de valor das revistas científicas. Mas contesta esta métrica, enfatizando que a mesma pode apresentar distorções por enfocar a avaliação das revistas científicas e não do artigo e do pesquisador, bem como servir de base para a concessão de cargos e salários, pois os pesquisadores ao publicarem seus manuscritos consideram o fator de impacto ao escolher os veículos de publicação. Nestes casos o fator de impacto é determinante para indicar a revistas científica em que será realizado a submissão de um determinado artigo. Yuret (2018) em concordância, defende que apesar de seus problemas, o fator de impacto das revistas científicas é a métrica de qualidade mais popular.

Seguindo Ortner (2010), afirma dizendo que geralmente o fator de impacto é uma medida objetiva da qualidade científica de uma revista. Destaca alguns fatores que podem influenciar no valor do fator de impacto: Fatores sociológicos: tipo de revista; número médio de autores por artigo; mês de publicação, bem como o fenômeno publicar ou perecer; e o fatores específicos: área de assunto da revista, tamanho da revista número de pesquisadores que trabalham no campo e o tipo de pesquisadores que trabalham no campo (indústria versus universidade). Além disso, o autor considera que o fator de impacto é uma medida estatística e, como tal, está correlacionado com o número de pesquisadores da área, visto que este número varia grandemente para vários campos da ciência. Leff (2005) também destaca o fator de impacto em seu estudo, em que ressalta que o fator de impacto, antes era uma ferramenta pouco conhecida, utilizada apenas pelos bibliotecários para escolher assinaturas para suas coleções e atualmente desenvolve grande poder sobre os rankings de classificação de revistas científicas.

Para Maity e Hatua (2016), a qualidade das revistas científicas é um fator muito importante a ser considerado, na publicação de artigos. Os autores contestam afirmando que todos nós procuramos uma revista de qualidade para submetermos nossos manuscritos, com o intuito de que eles sejam reconhecidos na ciência. Assim uma das técnicas utilizadas de antemão para medir a qualidade de uma revista é a taxa de aceitação sobre a taxa de rejeição, bem como calcular a frequência que os artigos de determinada revista são citados (Fator de Impacto). Esses indicadores de qualidade são muito bons, mas até onde sua eficácia é questionável? Os autores apresentam duas ferramentas disponíveis e recorrentes para a medição do fator de impacto: 0 Journal Citation Reports (JCR) e o Scimago Journals Rank (SJR). Estas ferramentas listam a classificação das revistas científicas, em especial as revistas disponíveis no banco de dados da Web of Science e Scopus da ISI. Há assim um questionamento em relação a como foi realizada a mediação do fator de impacto e o resultado da classificação das revistas. Como foram realizados os cálculos das citações dos artigos em revistas não europeias, russas, asiáticas e chinesas? 
Como são realizados a coletados dos dados dessas revistas que não são cobertas pelo Scopus ou Web of Science? Estas são questões aparecem como conflito quando se fala em fator de impacto como principal avaliar da qualidade das revistas científicas. Percebe-se assim que o fator de impacto não é a única ferramenta para medir a qualidade de uma revista científica, pode ser uma ferramenta subsidiária, que considere muitos outros fatores importantes para medir a qualidade de um periódico. Sendo assim é apresentado um modelo de avaliação da qualidade das revistas científicas, em que se pode medir uma revista a partir da sua própria decisão, sem depender de nenhum banco de dados comercial e decidir se a qualidade da revista selecionada é boa, muito boa, baixa ou média.

Winarko, Abrizah e Tahira, (2016) em seu estudo, assinalam o papel das revistas científicas na disseminação dos resultados de pesquisa, bem como, a importância da publicação científica no avanço dos estudos de pesquisadores. Partindo desse pressuposto, os autores discutem a questão da concentração das pesquisas acadêmicas, que em sua grande maioria concentram-se em apenas alguns países, considerados tecnologicamente e cientificamente avançados em relação aos outros países, visto que seu gasto com pesquisa e desenvolvimento é maior. Acreditam que deste modo, o mundo científico acaba sendo dividido em centros e periferias, que corresponde os estados afluentes e industrializados do hemisfério norte e os menos abastados e tecnologicamente menos avançados do Sul. Segundo os autores, a qualidade das revistas científicas nos países centrais é avaliada pela análise de citação, análise por pares, circulação, cobertura em indexação global ou serviços de abstração e muitos deles indexados por bancos de dados internacionais com a Web Of Science e o Scopus. Países como a Índia, o Irã e a China, também não se encontram na periferia, portanto têm seus próprios resumos e bancos de dados de citações. Avaliar o impacto das revistas científicas indonésias é considerado um desafio, pelo fato de estas revistas mão estarem facilmente disponíveis e a maioria delas é publicada em idioma nacional.

Shideler e Araújo (2016), em relação a qualidade das revistas científicas, salientam a influência do preço das assinaturas das revistas científicas de modo geral. Destacam que o fator de impacto é e ainda continua sendo o principal indicador de qualidade das revistas científicas, pois é a métrica mais conhecida e adotada pelas revistas. Mas em contrapartida, assume um papel crítico em publicações científicas, pois inclui a avaliação de promoções monetárias e empregatícias para pesquisadores que publicam em revistas de fator de impacto alto. Acreditam que seja necessário, utilizar novas metodologias para avaliar a qualidade relativa de uma revista científica. Inclui-se assim métricas como o Eingenfactor e o escore de influência do artigo. As pontuações do Eingenfactor juntamente com a popularidade do artigo ganharam visibilidade e passaram a ser relatados ao lado do fator de impacto como o Incites, métrica que mede $o$ 
número total de citações que uma reviste recebe em um ano, sem restrições quando estes artigos são publicados, muito semelhante ao fator de impacto. Essas métricas, estão sendo exploradas na literatura sobre como elas podem avaliar a qualidade de uma revista. No sentido mais amplo a qualidade de uma revista tem sido descrita em termos de "popularidade" ou em termos de "prestígio" da revista, ou seja, com que frequência ele é citado e aonde. Embora várias métricas de qualidade meçam atributos semelhantes em alguns casos, os indicadores são únicos da qualidade das revistas. Sendo assim, afirmam que a relação entre o preço e qualidade de uma revista é muito difícil de ser definida, pois pesquisas indicam que é muito percebido na comunidade acadêmica a semelhança entre o preço e qualidade de uma revista, ou seja, seu valor e qualidade andam na mesma direção. Contudo apontam que não está claro como as revistas científicas com alto número de artigos de revisão diferem em preço ou valor das revistas que se concentram todos ou principalmente na publicação de artigos de pesquisa originais.

Xu, Liu e Mingers (2015), discutem a avaliação das revistas científicas como um tema bastante intenso no estudo da bibliometria. Apresentam duas abordagens estudadas e aplicadas: revisão por pares, que é um questionário projetado para coletar opiniões sobre determinado estudo, abordagem muito utilizada para a classificação de uma série de revistas científicas; e o método bibliométrico, também conhecido como método quantitativo, dos quais o fator de impacto é o indicador mais conhecido e comumente utilizado para classificar revistas científicas. O fator de Impacto que calcula o número de citações de determinados documentos (artigos, resenhas e cartas) publicados em determinada revista no período de dois anos, também tem atraído atenção pelo fato de o banco de dados da Web of Science empregar este indicador. Mais recentemente, o índice $\mathrm{h}$ foi introduzido nesta lista, como um método de avaliação de pesquisa, que se estende em vários níveis da pesquisa, desde a avaliação dos pesquisadores individuais até as revistas científicas em si. É amplamente estudado na literatura, embora um índice-h possa ser calculado por qualquer pessoa, utilizando suas publicações, sua interpretação é mais clara utilizando o h-core, em que se procura identificar o núcleo mais produtivo da produção de um autor nas suas citações recebidas.

Duan; Nasri; Paknejad (2018), ressaltam que uma medida apropriada da qualidade das revistas científicas é essencial nas decisões de credenciamento, alocação de recursos, contratação, pagamento de mérito, posse e promoção em acadêmicos. Afirmam que a melhor prática atual para classificar a qualidade da revista científica é combinar a bibliometria com a avaliação de especialistas da área.

Chavarro, Ráfols e Tang (2018) salientam que a avaliação da pesquisa baseada na revista científica é uma prática amplamente adotada. Algumas avaliações de pesquisa usam a Web of Science (WoS) para identificar as revistas de "alta qualidade", que supostamente publicam 
excelentes pesquisas. A autoridade da WoS na qualidade da revista científica deriva de sua seleção de revistas de base em padrões editoriais e critérios de impacto científico. Estes podem ser considerados como critérios universalistas, o que significa que eles podem ser aplicados a qualquer revista, independentemente do seu local de publicação, idioma ou disciplina. Os autores concluem que o uso da WoS como uma ferramenta universalista para a avaliação da pesquisa pode prejudicar a ciência publicada em periódicos com padrões editoriais adequados e mérito científico.

Diamandis (2017) retrata os usos e o uso indevido do Journal Impact Factor (JIF) discutidos na literatura. Há alguns anos, previu-se que o JIF logo seria substituído. Nos últimos meses, os ataques ao JIF se intensificaram, com algumas organizações editoriais removendo gradualmente o indicador dos sites de seus periódicos. $O$ autor argumenta que a maioria, se não todos os usos indevidos do JIF estão relacionados ao seu nome. A palavra "impacto" deve ser removida, pois implica um atributo influente, seja para os periódicos, seus trabalhos publicados ou seus autores. O autor propõe, em vez disso, o uso de um novo nome, o "fator CAPCl", que significa a média da citação por item citado, que descreve com precisão o que é representado por essa medida.

Stumpf (2003), em seu estudo avaliou 26 revistas da área da Comunicação por meio da opinião de docentes/pesquisadores dos doze Programas de Pós-Graduação. Constituiu-se num estudo exploratório, com abordagem quantitativa, que utilizou como instrumento de coleta de dados um formulário próprio onde os sujeitos opinaram sobre os seguintes indicadores: avaliação geral da revista, sua contribuição para a área, prestígio junto à comunidade acadêmica, qualidade do conteúdo dos artigos, rigor na avaliação dos originais, regularidade da publicação, apresentação gráfica e distribuição.

Santos e Rabelo (2017) realizaram um estudo em que retratam através de uma revisão bibliográfica as fragilidades das publicações científicas nos dias atuais, bem como o impacto das publicações brasileiras a nível mundial. Os autores ressaltam a importância de se avaliar os periódicos científicos on-line como forma de manutenção da qualidade, e recomenda um formulário de avaliação para ele.

\section{Discussão dos dados}

Como pôde-se perceber, muito pouco ou quase nada se fala sobre a qualidade das revistas científicas em si. Discute-se muito sobre o fator de impacto das revistas científicas, um dos indicadores pelo qual pode ser medida a qualidade das revistas.

O fator de impacto é sem dúvidas o indicador mais mencionado e discutido em relação à avaliação da qualidade das revistas, que segundo Lee e Shin (2014) é sustentado pela 
suposição da frequência das citações que mede com precisão a importância de determinada revista para usuários finais. Outros indicadores também são utilizados, mas possuem menos visibilidade na comunidade científica, mas não os torna menos importantes, tal como o indicador proposto por Shideler e Araújo (2016), o Eingenfactor, que juntamente com a popularidade do artigo ganhou visibilidade por medir o número total de citações de uma revista em um ano, sem restrições de publicação e formato, semelhante no sentido de citações de artigos ao fator de impacto.

Vale ressaltar também a discussão proposta por Winarko, Abrizah e Tahira, (2016), que expõe a questão da concentração das pesquisas acadêmicas, que em sua grande maioria estão centradas nos grandes centros de pesquisa, ou seja, apenas em alguns países que possuem mais dinheiro para investir em pesquisas cientificamente avançadas. Acreditam que tal fato, acaba dividindo o mundo científico. A qualidade das revistas científicas dos países centrais, considerados os estados afluentes e industrializados do hemisfério norte, é realizada pela análise de citações, revisões por pares e outros indicadores de qualidade mais avançados e conhecidos. Países como o Irã, China e Índia possuem seus próprios bancos de citações, sendo assim a avaliação das revistas científicas nestes países é um grande desafio, por não estarem no mesmo parâmetro das revistas científicas dos países centrais.

Alguns aspectos de qualidade das revistas científicas são apontados por Carreli e Giannasi-Kaimen (2009). Estes aspectos são conhecidos como aspectos intrínsecos das revistas, que dizem respeito ao conteúdo da revista, que de acordo com Leite (2009) é "o principal determinante da qualidade da revista". Sobre os aspectos intrínsecos, podemos considerar que uma revista cumpra alguns critérios que podem qualificá-la, tais como, artigos originais na publicação de resultados de pesquisa e que tragam contribuição para o avanço do conhecimento e para a originalidade para a área do conhecimento, a revisão por pares, que diz respeito ao submissão do trabalho científico à especialistas da área e o corpo editorial da revista, que deve ser composto por pesquisadores reconhecidos na área do conhecimento específica da revista. Além dos aspectos apontados pelos autores, há também os aspectos extrínsecos das revistas, que dizem respeito ao formato de apresentação da revista científica, relacionados a periodicidade e pontualidade da revista.

Haddway et al. (2016), em seu estudo apontam como forma de validação da qualidade das revistas a presença o processo de revisão dos pares como uma importante garantia de qualidade de uma revista. Aspectos como pontualidade e periodicidade da revista também são extremamente importantes, pois dizem respeito ao comprometimento de determinada revista científica com seus prazos estabelecidos, quanto à resposta das submissões dos artigos à revista, bem como à publicação de suas edições. Uma revista científica de qualidade, não pode fechar 
submissões, limitar artigos ou escolher artigos para publicação, muito menos levar meses para responder a submissão de um artigo, fato que ocorre com muitas revistas que atendem aos indicadores de qualidade mais utilizados e conhecidos atualmente.

Rowlinson et al. (2015), reforçam a importância da revisão por pares nas revistas científicas quando fazem algumas críticas em relação à qualidade dos artigos das revistas, afirmando que os revisores especialistas das revistas científicas têm a capacidade de discernir os artigos de qualidade das revistas, não sendo necessária a consulta à métricas de citação ou até mesmo rankings de revista.

A qualidade das revistas científicas assim, engloba diversos aspectos que quando condensadas resultam em revistas científicas com padrões de qualidade, que atendem a comunidade científica, servindo desse modo como principal fonte de informações para a comunicação científica.

\section{Considerações finais}

Acreditamos que os poucos estudos sobre a temática se deve em grande parte, devido a sobrevalorização do fator de impacto, como também dos indicadores bibliométricos, que utilizam a contagem de citações como referência para publicação em revistas científicas. Elencamos aqui um dos principais fatores que podem influenciar nos estudos sobre a qualidade das revistas científicas, mas acreditamos que há também outros motivos pelos quais muito pouco se fala sobre o valor real da revista científica. Ao nosso ver muitos preferem "fechar os olhos" e continuar acreditando que a qualidade de uma revista se resume apenas em citações, e que quando mais uma revista traz prestígio próprio para o autor através de citações e mais citações, melhor será sua visibilidade na comunicação científica e mais prestígio terá.

Cabe aqui também mencionar que durante a extração dos artigos para a revisão, um dos artigos excluídos: Avaliação de periódicos científicos brasileiros da área da psicologia de Yamamoto et. al., menciona em seu resumo cinco conjuntos de itens que devem ser considerados na avaliação de uma revista científica, a saber: normalização, publicação, circulação, autoria, conteúdo e gestão pessoal. Mas em seu contexto menciona quase nada ou muito pouco sobre a qualidade de uma revista, não apresentando aspectos relevantes e claros sobre a qualidade das revistas científicas, fato que foi percebido em muitos dos artigos excluídos na seleção da amostra para a pesquisa, sendo desse modo excluídos por meio dos critérios de inclusão e exclusão propostos para a revisão sistemática em questão.

Vale ressaltar que esta pesquisa deve o intuito de trazer a discussão sobre a qualidade das revistas científicas para mais perto da realidade e principalmente instigar novas pesquisas 
sobre a temática, a fim de enriquecer os estudos e refletir sobre todos os aspectos que compõe a avaliação de uma revista científica de qualidade, meramente baseada em citações.

\section{Referências}

CARELLI, A. E.; GIANNASI-KAIMEN, M. J. Os periódicos científicos no compartilhamento da informação e do conhecimento: aspectos extrínsecos dos periódicos eletrônicos Qualis $A$ da área de ciência da informação. Encontros Bibli: Revista Eletrônica de Biblioteconomia e Ciência da Informação, v. 14, n. 27, p. 191-213, 2009.

CHAVARRO, Diego; RÀFOLS, Ismael; TANG, Puay. To what extent is inclusion in the Web of Science an indicator of journal 'quality'?. Research Evaluation, v. 27, n. 2, p. 106-118, 2018.

DIAMANDIS, Eleftherios P. The Journal Impact Factor is under attack-use the CAPCI factor instead. 2017. BMC Medicine, v. 15, n. 9, 2017. DOI 10.1186/s12916-016-0773-5

DUAN, Lian; NASRI, Farrokh; PAKNEJAD, Javad. A data-driven method for rating management information systems journals in the same scale of the Association of Business Schools Journal Guide. Expert Systems, v. 35, n. 6, p. e12309, 2018.

HADDAWY, Peter et al. A comprehensive examination of the relation of three citation-based journal metrics to expert judgment of journal quality. Journal of Informetrics, v. 10, n. 1, p. 162173, 2016.

KITCHENHAM, B.; CHARTERS, S. Guidelines for performing systematic literature reviews in software engineering. Technical Report EBSE 2007-001, Keele University and Durham University Joint Report, 2007.

KRZYZANOWSKI, Rosaly Favero; FERREIRA, Maria Cecília Gonzaga. Avaliação de periódicos científicos e técnicos brasileiros. Ciência da Informação, Brasília, v. 27, n. 2, p. 165-175, maio/ago. 1998.

LEE, Hakyeon; SHIN, Juneseuk. Measuring journal performance for multidisciplinary research: An efficiency perspective. Journal of Informetrics, v. 8, n. 1, p. 77-88, 2014.

LEFF, Donna. Making an impact: the rise of the impact factor as a measure of journal quality. Journal of the Academy of Nutrition and Dietetics, v. 105, n. 1, p. 29-30, 2005.

MAITY, Bapan Kumar; HATUA, Sudip Ranjan. Designing a model to evaluate scholarly publications with special reference to social sciences in India. Scientometrics, v. 109, n. 3, p. 2031-2048, 2016.

MINGERS, John; YANG, Liying. Evaluating journal quality: A review of journal citation indicators and ranking in business and management. European Journal of Operational Research, v. 257, n. 1, p. 323-337, 2017.

MIGLIOLI, S. Influência e limites do fator de impacto como métrica de avaliação na ciência. Ponto de Acesso, v. 11, n. 3, 2017. Disponível em: <http://www.brapci.inf.br/v/a/29249 >. Acesso em: 18 jun. 2018. 
ORTNER, Hugo M. The impact factor and other performance measures-much used with little knowledge about. International Journal of Refractory Metals and Hard Materials, v. 28, n. 5, p. 559-566, 2010.

ROWLINSON, Michael et al. Accounting for research quality: Research audits and the journal rankings debate. Critical Perspectives on Accounting, v. 26, p. 2-22, 2015.

SANTOS, Solange Maria dos. Perfil dos periódicos científicos de Ciências Sociais e de Humanidades: mapeamento das características extrínsecas. Tese de Doutorado. Universidade de São Paulo. Disponível em: <http://www.teses.usp.br/teses/disponiveis/27/27151/tde10112010-161748/en.php >. Acesso em: 16 out. 2018.

SANTOS, L. R.; RABELO, D. M. R. S. Produção científica: avaliação, ferramentas e indicadores de qualidade. Ponto de Acesso, v. 11, n. 2, p. 3-33, 2017.

SHIDELER, Geoffrey S.; ARAÚJO, Rafael J. Measures of scholarly journal quality are not universally applicable to determining value of advertised annual subscription price. Scientometrics, v. 107, n. 3, p. 963-973, 2016.

STUMPF, Ida Regina Chitto. Avaliação das revistas de comunicação pela comunidade acadêmica da área. Em Questão: revista da Faculdade de Biblioteconomia e Comunicação da UFRGS, v. 9, n. 1, p. 25-38, jan./junho 2003.

TARGINO, Maria das Graças; GARCIA, Joana Coeli Ribeiro. O editor e a revista científica: entre "o feijão e o sonho". In: FERREIRA, Sueli Mara Soares Pinto e TARGINO, Maria das Graças (org.). Mais sobre revistas científicas: em foco a gestão. São Paulo: Senac; Cengage Learning, 2008. p.41-72.

XU, Fang; LIU, WenBin; MINGERS, John. New journal classification methods based on the global h-index. Information Processing \& Management, v. 51, n. 2, p. 50-61, 2015.

WINARKO, Bambang; ABRIZAH, A.; TAHIRA, Muzammil. An assessment of quality, trustworthiness and usability of Indonesian agricultural science journals: stated preference versus revealed preference study. Scientometrics, v. 108, n. 1, p. 289-304, 2016.

YURET, Tolga. Author-weighted impact factor and reference return ratio: can we attain more equality among fields?. Scientometrics, v. 116, n. 3, p. 2097-2111, 2018. 